\title{
Factores mercadológicos utilizados por las organizaciones de producción cinematográfica mexicanas (OPCM) y su impacto en los ingresos en taquilla: caso de estudio Área Metropolitana de Nuevo León (Marketing factors used by the Mexican motion pictures production organizations, OPCM, and it's impact on box office revenues: case study in the Metropolitan Area of Nuevo Leon)
}

\section{David Fernando Lozano Treviño*, José Nicolás Barragán Codina*, Sergio Armando Guerra Moya*, Paula Villalpando Cadena*, María Eloísa Treviño Ayala* \& José Luis Pureco de León* \\ *Universidad Autónoma de Nuevo León, San Nicolás de los Garza, N.L., México, *Universidad de Monterrey, San Pedro Garza García, N.L. México \\ - Instituto Tecnológico y de Estudios Superiores de Monterrey, Monterrey, N.L., México, \\ Email: davidflozano@gmail.com}

Keywords: advertizing, availability in theaters, critics, pictures, recommendations, talent

\begin{abstract}
This document is intended to capture the marketing factors impact in the spectator purchase decisions of ticket at the box office in Monterrey and it metropolitan area, thereby increasing the income of the Mexican Motion Pictures Production Organizations (OPCM). Reference is made to the Mexican booming film industry of the 40's generating economic and cultural wealth. We analyze the recent performance of Mexican and U.S. cinema referencing to the years from 2002 to 2007 . We discuss some factors that can be used as supporting tools for marketing by de OPCM in their productions, such as special effects, enhancing the club effect, merchandising, genre and film classification, as well as the release date. We study in detail the marketing main factors used by the OPCM: advertising, critics, talent, recommendations and availability in theaters; and through the application of a poll and a linear regression analysis, we determine the impact of these on box office revenues.
\end{abstract}

Palabras clave: crítica, disponibilidad en taquilla, películas, publicidad, recomendaciones talento 
Resumen. El presente documento tiene como finalidad plasmar el impacto que tienen los factores mercadológicos en las decisiones de compra de un boleto en taquilla por parte de los espectadores de Monterrey y su área metropolitana; incrementando así los ingresos de las Organizaciones de Producción Cinematográficas Mexicanas (OPCM). Se hace referencia a la pujante industria cinematográfica mexicana de los años 40 generadora de riquezas económicas y culturales. Se analiza el desempeño reciente del cine mexicano y estadounidense tomando como referencia los años comprendidos del 2002 al 2007. Se comentan algunos factores que pueden emplearse como herramientas de apoyo al marketing por parte de las OPC en sus producciones, como lo son los efectos especiales, potenciar el efecto club, merchandising, la clasificación y el género del film y la fecha de estreno. Se estudian detalladamente los principales factores mercadológicos utilizados por las OPC: publicidad, crítica, talento, recomendaciones y disponibilidad en cartelera; y mediante la aplicación de una encuesta y un análisis de regresión lineal, se determina el impacto que éstos tienen en los ingresos en taquilla.

\section{Introducción}

El impulso de las Organizaciones de Producción Cinematográfica Mexicanas (OPCM) es importante en la búsqueda del crecimiento cultural y económico nacional. Son varios los factores que deben participar en conjunto como los artistas, el gobierno, los productores, inversionistas e instituciones educativas quienes con su trabajo y talento lograrán la meta antes mencionada.

La historia del cine mexicano cuenta con épocas interesantes en cuando a la generación económica y cultural, no sólo para el país sino también para otras naciones. En los años 40, la producción mexicana llegó a colocarse como el cuarto generador de ingresos para el país dentro del PIB (González, D. 2008). La tarea desempeñada por las OPCM mexicanas fue la de crear producciones que buscaban satisfacer a un mercado mundial necesitado de entretenimiento y de obras culturales ya que carecía de opciones debido a los enormes gastos incurridos durante la Segunda Guerra Mundial por los Aliados.

Debido al crecimiento de la industria cinematográfica en el país, las OPCM a su vez, lograron beneficiar a otras industrias como la minería mediante compuestos de plata (nitrato de plata, bromuro de plata, cloruro de plata y yoduro de plata) utilizados en la elaboración de rollos cinematográficos. También aportó al turismo gracias a los atractivos paisajes 
mexicanos que se mostraban en las películas por los que los espectadores internacionales mostraban interés. (Lozano, D. et al. 2009)

El impacto en las finanzas organizacionales que provocó el impulso de la producción cinematográfica fue factor clave en el crecimiento económico de la nación y su buena imagen en el extranjero, lo que fomentaba la producción y la comercialización de películas mexicanas en los Estados Unidos y en Europa y de esta forma obtener una utilidad atractiva para los inversionistas. (Lozano, D. et al. 2009)

Después de la Segunda Guerra Mundial, el descuido por parte del gobierno en la falta de estímulos a la industria y la escaza consideración de los productores en los gustos de los espectadores, provocó el decremento en ingresos y producción de la industria cinematográfica hasta llevarla a puntos críticos cercanos a la desaparición.

Las oficinas de gobierno han implementado una serie de estímulos que les permiten a las OPCM acceder a fuentes de financiamiento para levantar proyectos. Es importante comentar, que estos apoyos les permiten desarrollar proyectos de hasta 25 millones de pesos por película (www.imcine.gob.mx). La cifra es interesante, más escaza si no se usan esos recursos de forma inteligente y creativa.

También, para revivir este sector, los productores mexicanos deben trabajar en fórmulas cinematográficas y de negocios que permitan la creación de películas que sean atractivas para los espectadores en pantallas comerciales mexicanas, estadounidenses e internacionales, así como en buscar formas que no disparen los costos de producción ni que estén desfasados con los recursos con los que cuentan los estímulos gubernamentales y los inversionistas nacionales.

El eficiente destino a las partidas de mercadotecnia en los presupuestos, principalmente en sus áreas de publicidad (TV, radio, Internet, panorámicos, periódico, etc.), la calidad narrativa y visual que permitan generar una crítica y recomendaciones positivas, las negociaciones con los distribuidores que logren una amplia y duradera opción en cartelera para los espectadores y la contratación de talento hábil y atractivo para el espectador, pudieran ser clave para el incremento de los ingresos en taquillas y así generar riquezas para los productores y para la nación.

Este trabajo, se centrará en estudiar posibles factores mercadológicos causales de éxito en las producciones de cine que puedan ser aplicadas por 
las OPCM y que implementándolas les genere un incremento en los ingresos en taquillas, centrándonos en los Estados Unidos y pudiendo ser replicado en el resto del mundo.

\section{Planteamiento del Problema}

Debido a una insuficiente cantidad de estudios científicos que les permitan a los productores de cine mexicano conocer: qué factores mercadológicos aplicar en sus películas para incrementar las ventas en taquillas; este estudio busca demostrar si los factores aquí estudiados; publicidad, crítica, talento, recomendación y disponibilidad en cartelera, son causales de aumentos en los ingresos en taquilla al motivar a los espectadores a ver su producción.

\section{Objetivo}

Determinar si los factores mercadológicos utilizados en las producciones cinematográficas como los son la publicidad, la crítica, el talento, las recomendaciones y la disponibilidad en cartelera están relacionados con los ingresos en taquilla de las OPCM.

\section{Hipótesis general}

Los factores mercadológicos utilizados en las producciones cinematográficas: 1) publicidad, 2) crítica de expertos, 3) talento artístico, 4) recomendaciones de boca en boca y 5) disponibilidad en cartelera; están en función de los ingresos en taquilla de las OPCM al influir en las decisiones de compra de un boleto en taquilla por los espectadores.

\section{Visión de negocios y cultural en las organizaciones de producción cinematográficas (OPC)}

Todo productor o director de cine, debe estar interesado realmente en vivir de lo que piensa dedicarse. Éstos deben encontrar que unidos, el negocio y la cultura, lograrán una satisfactoria comercialización de sus películas. Además de que se crearán mayores fuentes de empleo y ellos 
mismos lograrán tener una cantidad importante de trabajo generador de riqueza, lo que beneficia la economía del país productor de cine. Por otro lado, habrá sin duda más fomento de la cultura, inspirativa y reflexiva, para los espectadores tanto nacionales como internacionales. (Lozano, D. et al, 2009). Así, es natural encontrar, en los empresarios cinematográficos con visión empresarial, una inquietud por la búsqueda de la rentabilidad y qué medios son los que le permitirán obtener riquezas económicas sin olvidar las culturalmente inherentes.

Los negocios de producción cinematográfica tienen dos vertientes importantes en cualquier país: 1) funcionan, por un lado, como motor industrial y de crecimiento económico y, 2) son un reflejo artístico y cultural, generalmente del país o países que producen la película (Gómez, R. 2005). Esto lo podemos palpar en los Estados Unidos, en un sentido económico, sus empresas son las más grande en cuanto a los ingresos captados en taquillas. En el 2008 generaron 28.1 mil millones de dólares (www.mpaa.org). Por el lado artístico, por medio del cine es que Estados Unidos ha llevado su cultura al resto del planeta unificándolo a sus deseos y valores (León, J. 2008).

Gráfica 1. Ingresos en taquilla del cine estadounidense en mercados domésticos e internacional

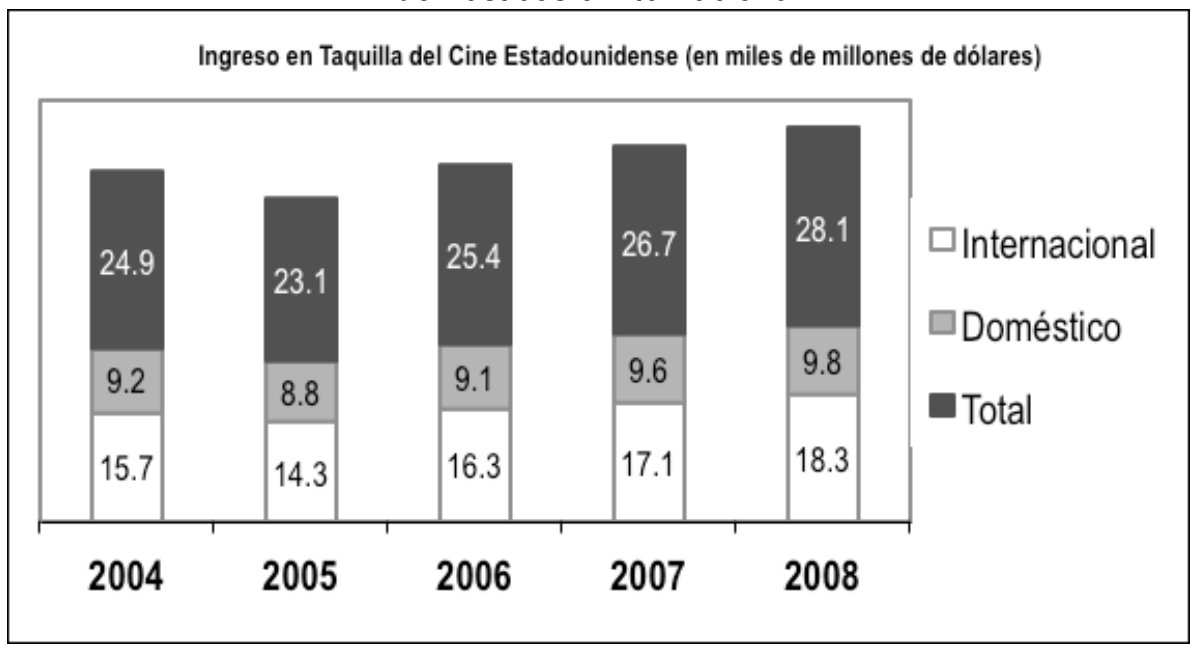

Fuente: MPAA Motion Picture American Asociation: http://www.mpaa.org 
Las OPCM dedicadas al cine llegaron a ser un motor económico y cultural del país ofreciendo productos para un mercado mundial que buscaba entretenerse. Durante los años de la Segunda Guerra Mundial, los negocios cinematográficos mexicanos, apoyados por los Estados Unidos, prosperaron de forma notable, gracias a que se enfocaban en satisfacer una demanda de diversión y arte fílmico que no podía ser ofrecida por las compañías estadounidenses ya que los recursos de aquella nación estaban destinados a la guerra. (Lozano, D. et al 2009) De 1941 a 1945, aproximadamente, el cine nacional conoció la que después fue llamada "Época de Oro".

En general, esta época ha sido recordada no sólo por los mexicanos a lo largo del tiempo, sino por todo el mundo, obteniendo un alto crédito por su calidad creativa y de marketing tanto en los Estados Unidos como en la Unión Europea, en donde hasta los espectadores de estos países todavía llevan en su mente a artistas como Cantinflas, Pedro Infante, Jorge Negrete, El Santo, María Félix, entre muchos otros.

Todas estas realizaciones complacieron al mercado, a los productores y hasta a los artistas más exigentes, logrando un equilibrio perfecto de negocio y arte que ahora no se presenta en el país. A principios del siglo la producción nacional era tan pobre que sólo 17 películas se hicieron en el año 2000, en el 2002 cayó a 7 films recuperándose, por de bajo de lo satisfactorio y de lo que necesitaría cualquier empresa para un crecimiento y desarrollo aceptable comercial y financieramente, con 42 y 41 películas en el 2005 y 2007 respectivamente (Gráfica 2), (www.imcine.gob).

El gobierno ha implementado diversos mecanismos que permitan generar una mayor producción de películas en el país, con el objetivo fundamental de apoyar las expresiones artísticas y buscar un crecimiento económico.

Hacia el sexenio de Vicente Fox, del 2000 al 2006, el gobierno comenzó a destinar recursos y a apostar en las empresas dedicadas a la producción cinematográfica. Impulsó el artículo 226 en la Ley del Impuesto Sobre la Renta (ISR), el cual permite a las empresas destinar hasta el 10\% de su ISR sin exceder 20 millones de pesos ni el $80 \%$ del total del costo de producción. Éste comienza a generar un número mayor de producciones y con mejor calidad, gracias a que las compañías encuentran fuentes de financiamiento que les permita, de alguna forma, competir con las películas 
extranjeras. Por otro lado, las empresas que otorgan el estímulo lo ven como una opción de publicidad gratis por medio de "product placment", créditos y menciones de sus productos o empresas dentro de las películas.

Gráfica 2. Películas mexicanas producidas apoyadas por el Estado de 2002 a 2007

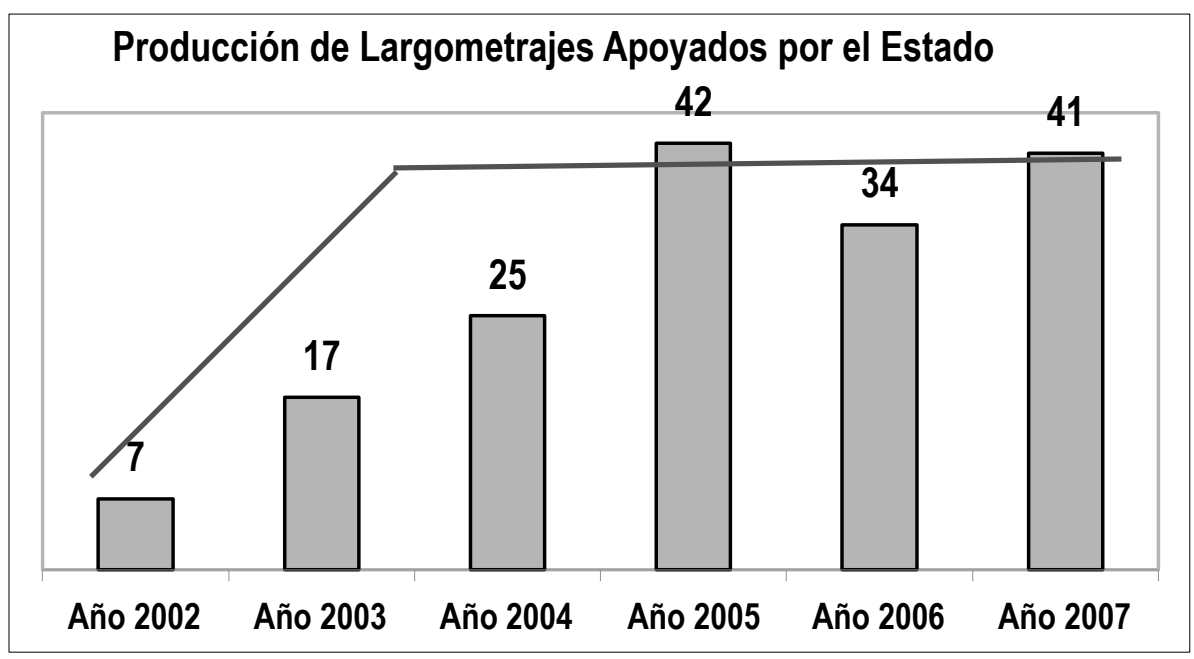

Fuente: IMCINE Instituto Mexicano de Cinematografía: http://www.imcine.gob

Por otro lado, es importante resaltar que estos estímulos no han sido suficientes para que el sector crezca, pues aunque ha habido una significativa producción del 2000 al 2007, por ejemplo, sólo 41 películas mexicanas se estrenaron en este último año (www.imcine.gob). A pesar del apoyo del gobierno a este sector, pareciera que las producciones todavía siguen siendo poco atractivas para los espectadores. Los empresarios de OPC deben tomar en cuenta diversos factores mercadológicos que les permitan vender una mayor cantidad de boletos de entrada al cine, éstos bien pudieran ser: altos niveles de publicidad, la crítica, el talento que participa en la producción, las recomendaciones de boca en boca o la disponibilidad en cartelera que les permita obtener atractivos ingresos.

Al final, los medios para financiarse se pueden encontrar de diferentes formas: buscar inversiones de los dueños de las OPCM, o de cualquier 
compañía que encuentre potencial financiero en el proyecto, mediante créditos otorgados por entidades dedicadas al préstamo o bien, con apoyos gubernamentales, como CONACULTA, CONARTE, Ayuntamientos, Gobiernos Estatales o Apoyos de la Federación (SHCP).

\section{Factores de apoyo mercadológicos a considerar en las producciones cinematográficas}

Aunque las OPCM no cuentan con los grandes presupuestos estadounidenses que les permiten tener mega producciones y destinar hasta el $40 \%$ de lo presupuestado a campañas de publicidad que logran persuadir al consumidor para que ven las películas (Miguel, J. 2004) entonces el reto de las producciones mexicanas será encontrar elementos que hagan que el producto sea interesante para el mercado. Las estrategias de apoyo al marketing son fundamentales para el éxito comercial de cualquier producto 0 servicio. Por lo tanto, es importante destinar el dinero del presupuesto de la forma más eficiente en donde su uso le permita a la producción maximizar el rendimiento por partida e impacto en los espectadores.

El cine como producto tiene algunos factores mercadológicos que podemos considerar para aumentar su éxito comercial tanto en México como allende las fronteras. Aplicado con creatividad, eficiencia y originalidad las OPCM pudieran atraer a la mayor cantidad posible de espectadores a las salas cinematográficas (Lozano, D. et al. 2010):

1. Efectos especiales.- aumentan el atractivo visual del producto y/o material artístico, además que genera nuevas investigaciones y el desarrollo de tecnologías.

2. Potenciar el efecto club.- es decir, mediante la creación de películas que busquen una franquicia (secuencia o saga), se generará una fidelidad de marca entre los espectadores.

3. Merchandising.- los ingresos no sólo se pueden obtener de la venta de la película, sino también con productos promocionales como juguetes, ropa, libros, guiones, etc.

4. Entre más costo menos el riesgo.- esto significa que si tenemos a un actor, director, productor o guionista de renombre, que por lo tanto incrementará los costos, el riesgo se reducirá al provocar que los 
espectadores sientan la confianza de que tendrán entretenimiento garantizado al ver el film.

5. Clasificación.- según ésta será la restricción del ingreso a las salas, aunque las películas con violencia $O$ violencia y sexo tienen más probabilidad de tener éxito. .- Una clasificación "A" estará destinada para todo público, mientras que una "C" excluye a los niños y adolecentes, por lo que reduce el mercado.

6. Género.- Encuestas efectuadas por la segunda exhibidora más importante del país, Cinépolis, los tres géneros preferidos en México son: Acción con el $44 \%$, Suspenso con el $14 \%$ y Comedia con el $13 \%$. (www.cinepolis.com) (Gráfica 3). Por lo tanto la realización nacional debe de ir encaminada a estos géneros que permitirán obtener mayores ingresos en las taquillas. Hay que considerar que estos gustos pudieran cambiar con los años, por lo que las empresas de producción cinematográficas deben realizar investigaciones de mercados que reflejen los deseos de los consumidores.

7. Fecha de estreno.- Si la película se lanza junto con "El Hombre Araña" o "Sreak" la competencia será bastante fuerte. Lo ideal será ser lanzada en el momento adecuado tanto por los estrenos que habrá ese día como por la temporada, por ejemplo, las películas con temáticas navideñas es preferible lanzarlas en los meses de noviembre o diciembre.

Ahora, lo anterior son factores que deben tomar los productores de las OPCM para lograr ampliar más sus estrategias de mercado y que a su vez le garantice mayores ingresos en taquillas. Por otro lado, es importante considerar que las estrategias mercadológicas en el cine se basan, al igual que las demás compañías que producen y comercializan bienes y servicios, en publicidad por diversos medios, las recomendaciones de boca en boca, la disponibilidad del producto en los puntos de venta, las recomendaciones de los expertos o líderes sociales (o crítica en el caso del cine) y el talento o el renombre de la empresa que los produce. Así, nuestro estudio se basa principalmente en cuál es el impacto que estas variables tienen en los ingresos en taquilla, para buscar, mejor planeación y ejecución de recursos financieros y creativos en dichos factores de marketing. 
Gráfica 3. Los 3 géneros preferidos por los espectadores mexicanos

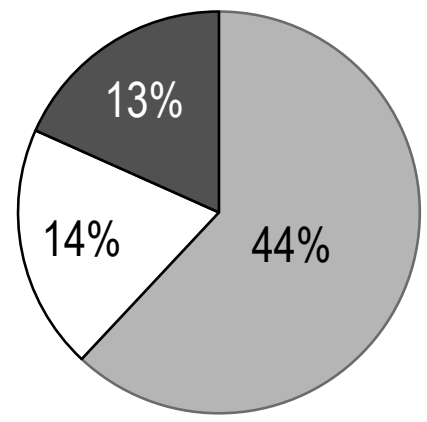

$\square$ Acción

$\square$ Suspenso

$\square$ Comedia

Fuente: Cinépolis: http://www.cinepolis.com

Principales factores mercadológicos implementados por OPCM mundiales en la búsqueda del éxito comercial

Estados Unidos y Europa han generado importantes recursos y un prospero crecimiento debido a las OPC. Los dueños en Estados Unidos, al producir películas, buscan también medios para distribuirlas haciéndose de compañías de cable y canales de televisión para así obtener mayores utilidades. General Electric es dueña de Universal Studios, NBC TV Network, CNBC, USA Bravo y SciFi Cable. Disney está comprendida por la productora Disney, Disney Channel y ABC Family Cable. Fox lo comprenden los estudios Fox, Fox TV Network, Fox programación por cable, etc. TimeWarner incluye a los estudios Warner y New Line, HBO y la programación por cable TBS. Sony está formada por los estudios Columbia, Tri-Star, ABC, CBS, NBC y Sony Channel, entre otras. Viacom es la productora Paramount, los estudios DreamWorks y los canales de tv por cable MTV y Nickelodeon. (Cusic, D. 2009).

En Europa podemos citar a Alemania y Austria y su cambio de perspectiva al buscar hacer negocio del cine. Los alemanes se han visto en mayor cantidad en pantallas internacionales. Su alta calidad y su introducción de elemento comercial mezclado con la parte cultural ha hacho a películas 
como "Corre Lola, Corre" (1998) de T. Tykwer, "La Caida del Halcón" (2005) de O. Hirschbiegel y "Good Bye Lenin!" (2003) de W. Becker obtener interesantes ingresos y premios. (Cookie, P. 2007). Los austriacos han destinado importantes recursos gubernamentales para aumentar su presencia en salas nacionales e internacionales. Las organizaciones de este país buscan diferenciarse y agregar atractivos mediante temas que sólo ellos pueden manejar con historias típicas de sus territorios involucrando anécdotas nacionales. Muchas veces se cuentan en inglés, sacrificando parte de su identidad cultural pero beneficiando los negocios internacionales. (Von Dassanowsky, R. 2005).

Algunas naciones, como México, adoptaron la fijación de cuotas para impulsar la producción cinematográfica de sus organizaciones en sus territorios. Esto a la larga no representó importantes beneficios. China, por ejemplo, provocó un enorme atraso al no permitir el ingreso de cine extranjero. (Lozano, D. et al. 2010) Los realizadores, quienes sólo buscaban el fin artístico, sin importarles la parte comercial, y que fueran apoyados por el gobierno, fracasaron cuando los privilegios gubernamentales fueron eliminados y se le dio un poco de más apertura al ingreso de películas extranjeras. Ahora estas empresas orientales deben tener una perspectiva más hacia el cliente y cómo persuadirlo para que adquiera un boleto de entrada al cine para ver su película. Sólo el cine que dejaba importantes rendimientos y que fue atractivo para realizar coproducciones con los Estados Unidos, principalmente, prosperó. (Kong, S. 2007). Los productores ubicaron a la comedia como el principal género en gustos de los espectadores chinos, aplicaron lenguajes y factores causales contemporáneos dejando atrás los mismos temas tocados en el pasado: la política y el drama, los cuales eran los impulsados por las esferas gubernamentales y que perecieron, cuando perecieron las compañias productoras que sólo buscaban un perfil político-artístico.

El menor control del Estado, resulta a la larga benéfico para la industria pues obliga a las organizaciones productoras a mantenerse actualizados y buscar ideas y productos atractivos para el mercado. Sólo los estímulos gubernamentales, y no su intervención total, impulsarán a un crecimiento y desarrollo adecuado. (Lozano, D. et al. 2010).

Existen varias observaciones que se le han hecho a la industria cinematográfica que es importante considerar al desear impulsar dicho sector 
en un ambiente de internacionalización. El cine es una industria riesgosa y a veces con pocos rendimientos. Muchas películas requieren de enormes presupuestos y lo que obtienen en taquilla puede ser similar a lo invertido. Es importante resaltar que el negocio no se hace sólo con la producción del película, sino también con su distribución, exhibición, merchandising, product placement y menciones dentro de la película, etc. y es por esto que un solo dueño es el comprende toda la cadena en Estados Unidos. (Lorenzen, M. 2007).

Pero, también es importante considerar que las estrategias de marketing que se vayan a emplear sean las adecuadas para lograr que la esencia principal de la OPC, la propia película, logre obtener atractivos ingresos y que lo proveniente del product placement, merchandising, etc. sea una ganancia adicional para los productores.

Otra observación importante es que, en un mundo globalizado, los mercados más grandes como son la India y China todavía mantienen cuotas al ingreso de producciones extranjeras y las películas que logran entrar se enfrentan a una fuerte competencia con la producción local, en muchos casos ya innovada. (Lorenzen, M. 2007). El mercado suele ser incierto en ocasiones y por lo tanto las estrategias mercadológicas para atraerlo deben ser innovadoras y persuasivas, por ejemplo, después de los atentados a las Torres gemelas, los espectadores no quisieron ver películas con temática dramática o trágica durante un largo periodo. Muchas producciones fueron retiradas de la cartelera y algunas ni siquiera lograron llegar a las salas: "Donnie Darko" (2001) de R. Kelly es una de ellas. La película contenía una producción atractiva, más la estrategia de marketing no logró llevarla siquiera a las salas de cine, siendo distribuida únicamente por DVD o TV de Cable. Factores atractivos como el talento encabezado por Drew Barrymore y la buena crítica no fueron suficientes.

Por otro lado, gracias a la globalización, es más común ver coproducciones entre países, lo que trae como consecuencia, a parte de un fácil acceso al mercado al no estar sujeto a las cuotas, mejores locaciones, mano de obra calificada, competitiva y conocedora de la región muchas veces a un precio más conveniente, ventajas fiscales, acceso a otras fuentes de financiamiento, división del riesgo entre una mayor cantidad de participantes, entre otros, que al final se traducirá en mayor comercialización y rentabilidad. (Morawetz, N. 2007). 
Resultado de la globalización es también el establecimiento de empresas del ramo en otros países diferentes a los de su lugar de origen. Lo que aumenta su presencia en estos mercados y logra entenderlos mejor para orientar la realización y promoción hacia los habitantes de la región. Por ejemplo, la película, "El Fin de los Tiempos" (2008) de N. Shyamalan, utilizó como estrategia posters con fondos con paisajes de las ciudades en donde se proyectó. En México se lanzaron cartelones con fondos del Cerro de la Silla de Nuevo León y el Ángel de la Independencia en la Ciudad de México para el Distrito Federal, mientras que en Estados Unidos se lanzó un poster con edificios y avenidas de Nueva York.

Con lo anterior, llegamos a considerar en este estudio, 5 herramientas de marketing que se utilizan en las producciones cinematográficas, pretendiendo encontrar cuáles de ellas impactan en los espectadores para efectúen un gasto en taquilla para ver una película, para que así, las OPCM enfoquen sus recursos presupuestarios y sus esfuerzos creativos en ellas:

1.- Publicidad.- Se refiere al mensaje sufragado por un patrocinador identificado y generalmente transmitido por algún medio de comunicación. Esta también puede ofrecer un incentivo extra para que el espectador adquiera un boleto en taquilla para su película, es decir, busca que los consumidores elijan su producción al resaltar los atributos de la misma (efectos especiales, talento, etc.). La publicidad se puede llevar por diferentes canales: TV, Internet, periódicos, revistas, radio, etc. (Russell, T. 2001). Recordemos que entre mayor sea la publicidad, más persuasión se ejerce en el espectador para que vaya a ver la película.

La publicidad en cines se construye en el superlativo recurrente: la mejor película, nunca antes vista, nunca antes, de todos los tiempos, etc. Las campañas publicitarias se disputan los adjetivos: emocionante, enternecedor, sorprendente, excitante, estremecedor. De modo invariable se busca llegar a los sentimientos y sensaciones del público. Al espectador de edad mediana, la publicidad le mostrará el sentimiento de la fuga; al niño le mostrará un oasis prometido; al anciano uno de la memoria. (González, D. 2008).

Al momento de tomar una decisión para ver una película, los espectadores toman más en cuenta los cortos de la película (Eliashberg, J. 1997). En ellos encontrarán los momentos más emocionantes y atractivos, así como de la temática a tratar, el género e incluso los actores, directores y 
productores además de, probablemente, comentarios que hayan hecho los críticos de cine.

Otra herramienta utilizada para publicitar la película son los posters, los cuales reflejan una parte atractiva del film y están diseñados adecuadamente para que atrapen la atención del espectador rápidamente y los motiven a comprar un boleto para ver la película. Igualmente mostrar los nombres de los participantes suele ser un atractivo extra.

Los presupuestos de las películas llegan a destinar hasta el $40 \%$ a la publicidad. Como se mencionó antes, esta estrategia permite mostrar lo mejor de los films para que se motiven a verlos. Estos gastos, junto con los de producción, son los más altos en el negocio, pero también son los que aumentan más la venta de boletos en taquilla.

2.- Crítica.- Según el portal de la Escuela de Crítica Cinematográfica (www.aulacritica.com), la crítica es un juicio sobre una película emitido por una persona con conocimientos del lenguaje cinematográfico y su historia. Estas opiniones sobre los films son generalmente externados en periódicos, revistas, radio, televisión e Internet y pudieran impactar en los espectadores que necesiten mayor información para tomar decisiones sobre si ver o no ver cierta película. E igualmente es más importante durante los primeros días del estreno, ya que los clientes todavía no obtienen información boca a boca de otros espectadores. (Eliashberg, J. 1997).

Siempre será importante que las producciones obtengan una buena crítica por parte de la prensa, ya que reflejará la calidad del film. Es notorio que el crecimiento del cine a nivel global es enorme, gracias en parte a toda la publicidad que se genera y a los reflectores que capta por parte de las críticas. Así, los estudios están cada vez más enfocados en realizar investigaciones de mercado para encontrar lo que los espectadores quieren ver en el cine, darles lo que buscan y de esta forma agradar igualmente a los críticos, quienes por lo general no son expertos cinematográficos.

Pareciera que la crítica no es determinante para los espectadores para ver una película o no pero aun así es incuestionable que los estudios busquen tener buenas relaciones con los críticos, ya que, aunque pareciera que no influyeran mucho, es conveniente tener una relación positiva con quien será uno de los primeros informantes del producto audiovisual.

3.- Talento.-Es el equipo humano profesional compuesto en su mayoría por expertos dedicados al espectáculo y entretenimiento de un 
público. (www.talentoartistico.com.mx) Es sin duda el recurso más importante en una película. Está compuesto por el personal que está frente a las cámaras: actores principales, de reparto, extras y dobles y por el crew detrás de cámaras: director, productor, guionista, músico, fotógrafo, camarógrafos, etc. Por lo tanto, propiamente son los que elaboran el producto y se encargan de su atractivo y calidad. Muchas personas piensan que el éxito de una película está garantizado con buenos actores, directores, guionistas y/o productores mas ha habido numerosas películas que fracasaron con estrellas o productores reconocidos.

Por supuesto que es fundamental que la película cuente con productores experimentados y creativos para que el éxito esté prácticamente asegurado. (Brunet, J. 2004). Siendo la idea, probablemente lo más importante, la buena ejecución de ésta estará dada por talento capacitado, experimentado y creativo, mismo que deberá pensar en forma global y en todas las faces de una película: idea, desarrollo, pre-producción, producción, post-producción y distribución.

4.- Recomendaciones.- Es propiamente la publicidad de boca a boca, aquella que consiste en pasar información por medios verbales, especialmente recomendaciones de una manera personal. (www.fundeu.es) Si la película es buena, esta publicidad pudiera ser muy importante durante los primeros días de exhibición de una película. Los espectadores deciden, principalmente, ver una película con base en las recomendaciones de boca en boca 0 sus experiencias pasadas. (De Vany, A. 2004). Este comportamiento se da como en cualquier producto o servicio siendo los clientes anteriores los principales medios publicitarios que pudieran ser utilizados por las OPCM.

5.- Disponibilidad en cartelera.- Se refiere a la exhibición de una película en el momento en que el espectador va al complejo cinematográfico. Este factor pudiera intervenir cuando el espectador asiste al cine si saber qué película ver, sino más bien tomará la decisión según lo que encuentre en cartelera.

Ahora bien, lo complejo puede ser: cómo lograr obtener salas de exhibición. Negociando y produciendo películas que entretengan a los espectadores.

Los distribuidores hacen contratos con los productores sobre el reparto de ganancias variando según los ingresos en taquilla y el éxito de la película. 
(De Vany, A. 2004). Por otra parte, los exhibidores buscan mantener una variedad de películas en las salas que incluyan películas comerciales, de cuotas gubernamentales y de cine de arte. (Rosen, S. 2003). Igualmente es importante considerar que hay países que tienen restricciones sobre cierto contenido de las películas.

Un ejemplo de producción lo pudiera dar China. Sus organizaciones han llevado acabo diversos benchmarking de organizaciones estadounidenses, que al producir películas que los espectadores quieren ver en pantalla, copian ese modelo buscando llevar más espectadores a las pantallas cinematográficas algo que sin duda motivara a os distribuidores de cine.

Otro caso de benchmarking lo hace Nigeria y su Nollywood, siendo el tercer productor más grande de películas en el mundo. Lo genial aquí es que sólo tomaron el modelo heroico, muy demandado, y lo adaptaron a la sociedad africana, plasmando el ambiente de Lagos, la extrema inseguridad y la pobreza. (Haynes, J. 2007). Estas películas infunden deseos o temores de los espectadores, lo que los hace asistir a las salas, o adquirir sus películas, lo que incentiva a los distribuidores a trabajar en llevar estas películas a las carteleras cinematográficas.

El $80 \%$ de los espectadores en países como Alemania o México, prefieren ver películas con la estructura hollywoodense, por lo que los benchmarking parece que pudiera garantizar disponibilidad en cartelera.

Por último, la distribución y la exhibición son los que cierran el negocio principal del cine. El primero es el que negocio con el segundo la cantidad de copias que se van a lanzar y que deberán ser exhibidas, con él también se negocia parte de la publicidad y cómo será la distribución de las ganancias entre el exhibidor y el distribuidor. La exhibidora será la encargada de dar el servicio de ver la película, con audio de alta calidad, buena imagen, etc. además es el que tiene la decisión de proyectar la película en un determinado número de salas.

\section{Recomendaciones de marketing para las OPCM en expansión}

Algunas recomendaciones para aumentar la comercialización y hacerlo más productivo, se dirigen a (Fernández, V. 2008): 
- Buscar coporducciones con Hollywood

- Crear películas en las que las grandes corporaciones no estén interesadas hacer.

- Buscar darle al espectador lo que demanda.

- Pensar en películas que hagan reflexionar al espectador.

- Reflejar el folklor puede beneficiar aunque aumenta el riesgo de éxito.

Algunos factores que ponen en desventaja al cine nacional son hacia una competitividad en los negocios como:

- La excesiva promoción y publicidad que se les hace a las producciones hollywoodenses.

- La poca aceptación que se le da a las distribuidoras independientes.

- Los gustos tan homogéneos que ya se establecieron en el mercado.

- El alto riesgo que impera en la distribución que hace necesario contar con un flujo continuo y regular de películas con semejante calidad.

- Las economías de escala.

Además, complementando lo que se ha mencionado, las empresas de producción cinematográfica mexicanas deben trabajar también en un concepto duradero de marketing en las películas. La lealtad que se genere en el espectador es un factor clave para alargar la vida del concepto y obtener grandiosos beneficios económicos. Para esto existen los conceptos de saga y series.

Las series cinematográficas reducen la incertidumbre sobre el éxito. Las series comparten con las películas no seriadas varias pautas de comportamiento tendientes a asegurar el éxito: actores conocidos, directores, altos presupuestos, utilizan mecanismos específicos para el merchandising y la asociación de marcas. (Miguel, J. 2004).

El merchandising debe ser visto como una actividad tendiente a aumentar la rentabilidad, a la vez que nos da publicidad. Es un elemento que crea fidelidad entre los consumidores.

La saga es un relato largo y detallado, mientras que la serie es un conjunto de capítulos, frecuentemente con independencia argumental, pero con los mismos personajes básicos. Entre las sagas pudiéramos mencionar "El Señor de los Anillos de P. Jackson y "Matrix" de los hermanos Wachowski. En las series están "James Bond" e "Indiana Jones". 
Por último, conocer el impacto que tiene la publicidad, la crítica, el talento que participa en una película, las recomendaciones y la disponibilidad en cartelera, en el espectador con el fin de que decida adquirir un boleto en taquilla, le permitirán a las OPCM enfocarse en la estrategia de marketing adecuada que le lleven a mayores ingresos en taquillas.

\section{Diseño del instrumento}

Se aplicó una encuesta con el objetivo de conocer qué factores mercadológicos motivan a los espectadores en la compra de un boleto en taquilla para ver una película. Las preguntas se respondieron con base en una escala del 1 al 10, en donde 1 es "Nada" y 10 "Totalmente"

La cuestión aquí analizada hace referencia a: De los siguientes aspectos, ¿cuánto influyeron en ti, el último mes, para que veas una película? Dentro de las opciones que debió contestar el encuestado encontramos las variables aquí estudiadas: Publicidad, Crítica, Talento, Recomendaciones y Disponibilidad en cartelera. Igualmente se le preguntó a los espectadores: ¿Cuánto gastas mensualmente, sólo en ti, en boletos del cine.

Metodología de la Investigación

Para conocer los factores que influyen en los espectadores para la decisión de compra de un boleto en taquilla, se selecciono una muestra en el área metropolitana de Monterrey, una ciudad cosmopolita con características, en cuanto a sus deseos cinematográficos, al menos, muy parecidos a los de los espectadores occidentales. Las características estadísticas de la población, de la cual se obtuvo la muestra, fue la siguiente:

- Parte de los datos mostrados fueron obtenidos de la página del Inegi (www.inegi.gob.mx) y de Imcine (www.imcine.gob.mx)

- La población en Nuevo León es de 4,653,458 para el 2010

- El $88 \%$ de los habitantes viven en el área metropolitana, es decir, $4,095,043$.

- Del total de la población del área metropolitana, el $70.6 \%$ tiene 15 años o más, los cuales fueron considerados para contestar la encuesta, debido al desarrollo en la apreciación cinematográfica que tienen. El resultado es de $2,891,100$. 
Utilizando los parámetros anteriores se procedió a determinar el tamaño de la muestra adecuado considerando un error de estimación (E) del $10 \%$ y un grado de confianza del $95 \%$.

$E$ (Error de estimación $)=10 \%, p=0.5, q=0.5, N$ (Población $)=2,891,107$. Con un $95 \%$ de confianza

Con lo anterior obtenemos que:

$$
\begin{gathered}
n=\left(z^{2} p q\right) / E^{2} \\
n=\left(1.96^{2} \times 0.5 \times 0.5\right) \div(0.1)^{2} \\
n=96
\end{gathered}
$$

Cercano a los 100

La encuesta se aplicó a 100 espectadores tratando de ser heterogéneos en su aplicación, tendiendo a la misma cantidad para hombres y mujeres y diversidad de edades.

Para asegurar que las encuestas fueran aplicadas en diferentes estratos económicos, se ubicaron las principales cadenas cinematográficas del estado de Nuevo León, Cinépolis, Cinemex (MMCinemas) y Cinemark, las cuales se separaron según niveles socioeconómicos, para lo que se consideró los precios en taquilla publicados en sus páginas de Internet. (www.cinepolis.com) (www.cinemex.com) (www.cinemark.com).

Para determinar el estrato económico al que se aplicó la encuesta se realizó la siguiente clasificación (Tabla 1):

Tabla 1. Determinación del estrato económico al que se le aplicó la encuesta

\begin{tabular}{ll}
\hline Estrato Socioeconómico & Costo del Boleto en Pesos Mexicanos \\
\hline Bajo & Menor a 35 \\
Medio & Mayor o igual a 35 y menor o igual a 60 \\
Alto & Mayor a 60 \\
\hline
\end{tabular}

Se aplicó 7 encuestas por cine. La selección de los cines en donde se aplicará la encuesta se hizo mediante un sorteo, en los que se consideraron los 39 complejos cinematográficos, obteniendo como resultado los marcados con asterisco. (Tablas 2, 3 y 4). 
Tabla 2. Cines seleccionados y cantidades de encuestas a aplicar por cine (Alta)

\begin{tabular}{lcc}
\hline Complejo & Precio & Precio Niño \\
\hline *Galerías Monterrev VIP - Cinébolis & $\$ 111$ & $\$ 111$ \\
Galerías Monterrey - Cinépolis & $\$ 64$ & $\$ 55$ \\
Galerías Valle Oriente VIP - Cinépolis & $\$ 115$ & $\$ 111$ \\
Galerías Valle Oriente - Cinépolis & $\$ 64$ & $\$ 55$ \\
*Humberto Lobo - Cinemex & $\$ 66$ & $\$ 51$ \\
San Pedro - Cinemex & $\$ 110$ & $\$ 110$ \\
*San Agustín - Cinemex & $\$ 66$ & $\$ 51$ \\
Leones (MMCinemas) & $\$ 61$ & $\$ 49$ \\
Promedio & $\$ 82$ & $\$ 74$ \\
\hline Nota 1. Total de cines: & 8 & \\
Nota 2. Presentación en \%: & $21 \%$ & 20 \\
Nota 3. Proporción de la muestra: & 20.51 & \\
Nota 4. Propuesta de tamaño de la muestra: & en 3 cines $\left(^{*}\right), 7$ encuestas por cine
\end{tabular}

Tabla 3. Cines seleccionados y cantidades de encuestas a aplicar por cine (Media)

\begin{tabular}{lcc}
\hline Complejo & Precio & Precio Niño \\
\hline${ }^{*}$ Citadel - Cinébolis & $\$ 51$ & $\$ 38$ \\
${ }^{*}$ Cumbres Mty - Cinépolis & $\$ 54$ & $\$ 45$ \\
Garza Sada - Cinépolis & $\$ 60$ & $\$ 46$ \\
Sendero Lincoln - Cinépolis & $\$ 35$ & $\$ 35$ \\
${ }^{*}$ Sendero Monterrey - Cinépolis & $\$ 39$ & $\$ 39$ \\
Céntrika - Cinemex & $\$ 55$ & $\$ 44$ \\
${ }^{*}$ Cumbres - Cinemex & $\$ 39$ & $\$ 36$ \\
Revolución - Cinemex & $\$ 56$ & $\$ 46$ \\
Linda Vista - Cinemex & $\$ 55$ & $\$ 44$ \\
Fiesta Anáhuac - Cinemex & $\$ 55$ & $\$ 44$ \\
Las Plazas Outlet - Cinemex & $\$ 40$ & $\$ 34$ \\
San Nicolás - Cinemex & $\$ 35$ & $\$ 31$ \\
Adana Lincoln - Cinépolis & $\$ 35$ & $\$ 35$ \\
*La Silla (MMCinemas) & $\$ 56$ & $\$ 46$ \\
Lincoln (MMCinemas) & $\$ 38$ & $\$ 35$ \\
Las Américas (MMCinemas) & $\$ 48$ & $\$ 40$ \\
Santo Dominao (MMCinemas) & $\$ 37$ & $\$ 30$ \\
*Santa Catarina - Cinemex & $\$ 57$ & $\$ 47$ \\
Concordia - Cinemex & $\$ 37$ & $\$ 30$ \\
*Plaza La Fe - Cinemark & $\$ 45$ & $\$ 33$ \\
Plaza Real - Cinemark & $\$ 51$ & $\$ 39$ \\
Promedio & $\$ 47$ & $\$ 39$ \\
\hline Nota 1. Total de cines: & 21 & \\
Nota 2. Presentación en $\%:$ & $54 \%$ & 54 \\
Nota 3. Proporción de la muestra: & 53.85 & \\
Nota 4. Propuesta de tamaño de la muestra: & en 7 cines( $\left.{ }^{*}\right), 7$ encuestas por cine
\end{tabular}


Tabla 4. Cines seleccionados y cantidades de encuestas a aplicar por cine (Baja)

\begin{tabular}{lcc}
\hline Complejo & Precio & Precio Niño \\
\hline *Interplaza - Cinépolis & $\$ 25$ & $\$ 25$ \\
*Pablo Livas - Cinépolis & $\$ 25$ & $\$ 25$ \\
San Roque - Cinépolis & $\$ 29$ & $\$ 29$ \\
Sendero Apodaca - Cinépolis & $\$ 30$ & $\$ 30$ \\
Valle Soleado - Cinépolis & $\$ 25$ & $\$ 25$ \\
Multicinemas Los Ángeles - Cinépolis & $\$ 25$ & $\$ 17$ \\
Sun Mall Guadalupe (MMCinemas) & $\$ 33$ & $\$ 32$ \\
*Escobedo (MMCinemas) & $\$ 31$ & $\$ 30$ \\
Sun Mall Juárez - Cinemex & $\$ 28$ & $\$ 27$ \\
${ }^{*}$ Contry (MMCinemas) & $\$ 31$ & $\$ 30$ \\
Promedio & $\$ 28$ & $\$ 27$ \\
\hline Nota 1. Total de cines: & 21 & \\
Nota 2. Presentación en \%: & $54 \%$ & 54 \\
Nota 3. Proporción de la muestra: & 53.85 & \\
Nota 4. Propuesta de tamaño de la muestra: & \multicolumn{2}{c}{ en 7 cines $\left({ }^{*}\right), 7$ encuestas por cine }
\end{tabular}

\section{Resultados de la Investigación}

Se pidió a los espectadores considerar el mes anterior al corriente para contestar la encuesta. La pregunta hace referencia a "De los siguientes aspectos, ¿cuánto influyeron en ti, el último mes, para que veas una película?". Los aspectos a considerar fueron:

- Publicidad (X1).- es decir el marketing hecho por la producción para promocionar la película en diferentes medios: panorámicos, comerciales de radio, televisión, prensa y/o Internet, etc.

- Crítica (X2).- según la Real Academia de la Lengua Española, es la expresión pública sobre una película. Por lo tanto, si fue considerada buena o mala por expertos apreciadores de películas.

- Talento (X3).- ya sea los productores, directores, guionistas o algún miembro artístico detrás de cámaras o bien, actores principales 0 de reparto.

- Recomendaciones (X4).- alguna opinión favorable o desfavorable hecha por familiares o conocidos que hayan visto previamente la película, quienes influyen en que el encuestado vea o un algún film. 
- Disponibilidad en cartelera (X5).- es decir, las opciones existentes que hay en los complejos cinematográficos.

Dichos aspectos fueron considerados como variables independientes siendo respondidos con base en una escala del 1 al 10, en donde 1 es NADA y 10 TOTALMENTE. Por otro lado, como variable dependiente se consideró:

- Gasto mensual por espectador (y).- es la erogación que hace el espectador en boletos de cine en un mes.

Lo siguiente se analizó mediante una regresión lineal:

Donde:

$$
y=\beta 0+\beta 1 X 1+\beta 2 X 2+\beta 3 X 3+\beta 4 X 4+\beta 5 X 5+e
$$

$\mathrm{X} 1=$ Publicidad

$\mathrm{X} 2=$ Crítica

$\mathrm{X} 3=$ Talento

$\mathrm{X} 4=$ Recomendaciones

X5 = Disponibilidad en cartelera

$\mathrm{y}=$ Gasto mensual en taquilla

En la Tabla 3 se presenta la descripción de la muestra a la que se le aplicó la muestra.

Tabla 3. Descripción de la muestra a la que se le aplicó la encuesta sobre factores causales del gasto en taquilla. Elaborado en SPSS

\begin{tabular}{rccccc}
\hline & Frecuencia & Porcentaje & $\begin{array}{c}\text { Porcentaje } \\
\text { válido }\end{array}$ & $\begin{array}{c}\text { Porcentaje } \\
\text { acumulado }\end{array}$ \\
\hline Válidos & 1 & 49 & 48.5 & 48.5 & 48.5 \\
& 2 & 52 & 51.5 & 51.5 & 100.0 \\
Total & 101 & 100.0 & 100.0 & \\
\hline
\end{tabular}

En donde 1 representa las personas encuestadas del sexo masculino, mientras que 2 a las del sexo femenino. La asimetría obtenida es de -0.06, lo cual es aceptable. 
Para las edades se logró observar una variedad de encuestados bastante amplia, desde los 15 años, edad en donde los espectadores ya pudieran emitir un juicio más racional, hasta los 67 . Los resultados obtenidos fueron los siguientes:

Tabla 4. Estadístico descriptivo para, según los espectadores, cuánto influyen los siguientes factores en la compra de un boleto en taquilla. Corrido en

\begin{tabular}{lccc}
\multicolumn{4}{c}{ SPSS } \\
\hline & Media & Desviación típ. & $\mathbf{N}$ \\
\hline GASTO & 420.2475 & 289.38070 & 101 \\
PUBLICIDAD & 7.0990 & 2.76226 & 101 \\
CRITICA & 5.6535 & 3.27547 & 101 \\
ARTISTA & 7.2574 & 2.93139 & 101 \\
RECOMENDACIONES & 7.6634 & 2.52696 & 101 \\
CARTELERA & 7.2376 & 2.54617 & 101 \\
\hline
\end{tabular}

En la Tabla 4 observamos, que salvo la variable Crítica, el resto de las variables independientes tienen su media en valores entre 7 y 8 (en un rango de 1 a 10). Di igual manera, la desviación estándar para todas las variables que no son la Crítica tienen una desviación estándar en un rango de 2.5 y 3.0.

La Tabla 5 muestra la correlación de Pearson que hay entre variables independientes y la variable dependiente. Vemos que solamente un par de variables independientes tienen alta correlación: la publicidad y las recomendaciones (0.538), más el gasto con ninguna tiene una correlación significativa. La correlación es entre variables independientes para observar la intensidad del impacto entre ellas. Aquí traen correlaciones bajas lo que implica que las variables son independientes y ayuda a utilizar la regresión por la independencia de las variables, es importante que las variables sean independientes entre si, ya que de no ser así implicaría que la presencia de alguna de ellas puede explicar el comportamiento de otra en lugar de explicar el comportamiento de la variable dependiente, afectando de manera significativa la capacidad predictiva del modelo. Hay muchos niveles de significancia bastante aceptables como los de .000 ( un nivel de significancia debe estar debajo de .005) 
Tabla 5. Cuadro de correlación entre variables. Corrido en SPSS

\begin{tabular}{|c|c|c|c|c|c|c|c|}
\hline & & GASTO & PUBLICIDAD & CRÍTICA & ARTISTAS & RECOMENDACIONES & CARTELERA \\
\hline \multirow{6}{*}{ 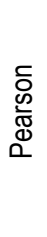 } & GASTO & 1.000 & 0.129 & 0.070 & 0.176 & -0.031 & -0.069 \\
\hline & PUBLICIDAD & 0.129 & 1.000 & 0.372 & 0.256 & 0.538 & 0.226 \\
\hline & CRÍTICA & 0.070 & 0.372 & 1.000 & 0.391 & 0.412 & 0.066 \\
\hline & ARTISTAS & 0.176 & 0.256 & 0.391 & 1.000 & 0.239 & 0.178 \\
\hline & RECOMENDACIONES & -0.310 & 0.538 & 0.412 & 0.239 & 1.000 & 0.264 \\
\hline & CARTELERA & 0.069 & 0.226 & 0.066 & 0.178 & 0.264 & 1.000 \\
\hline \multirow{6}{*}{ 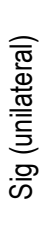 } & GASTO & . & 0.100 & 0.245 & 0.039 & 0.377 & 0.246 \\
\hline & PUBLICIDAD & 0.100 & & 0.000 & 0.005 & 0.000 & 0.012 \\
\hline & CRÍTICA & 0.245 & 0.000 & . & 0.000 & 0.000 & 0.255 \\
\hline & ARTISTAS & 0.039 & 0.005 & 0.000 & . & 0.008 & 0.083 \\
\hline & RECOMENDACIONES & 0.377 & 0.000 & 0.000 & 0.008 & & 0.004 \\
\hline & CARTELERA & 0.246 & 0.012 & 0.255 & 0.038 & 0.004 & 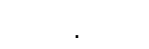 \\
\hline \multirow{6}{*}{$z$} & GASTO & 101 & 101 & 101 & 101 & 101 & 101 \\
\hline & PUBLICIDAD & 101 & 101 & 101 & 101 & 101 & 101 \\
\hline & CRÍTICA & 101 & 101 & 101 & 101 & 101 & 101 \\
\hline & ARTISTAS & 101 & 101 & 101 & 101 & 101 & 101 \\
\hline & RECOMENDACIONES & 101 & 101 & 101 & 101 & 101 & 101 \\
\hline & CARTELERA & 101 & 101 & 101 & 101 & 101 & 101 \\
\hline
\end{tabular}

Por medio de la $R$ cuadrado corregida certificamos que el 0.01 de la variabilidad en " $y$ " está explicada por este modelo de regresión (Tabla 6). Al igual que por medio del Durbin-Watson (1.807) corroboramos que el modelo tiene suficiencia estadística para poder medir la relación entre las variables.

Tabla 6. Cambio de la variable dependiente por las variables predictoras. Corrido en SPSS. Estadísticos de Cambio

\begin{tabular}{|c|c|c|c|c|c|c|c|c|c|}
\hline R & $\begin{array}{c}\mathbf{R} \\
\text { cuadrado }\end{array}$ & $\begin{array}{l}\text { R cuadrado } \\
\text { corregida }\end{array}$ & $\begin{array}{c}\text { Error típ. } \\
\text { de la } \\
\text { estimación }\end{array}$ & $\begin{array}{c}\text { Cambio } \\
\text { en } \mathrm{R} \\
\text { cuadrado } \\
\end{array}$ & $\begin{array}{c}\text { Cambio } \\
\text { F }\end{array}$ & gl1 & gl2 & $\begin{array}{c}\text { Sig. del } \\
\text { cambio } \\
\text { en F } \\
\end{array}$ & $\begin{array}{l}\text { Durbin- } \\
\text { Watson }\end{array}$ \\
\hline .245 & .060 & .010 & 287.86487 & .060 & 1.211 & 5 & 95 & .310 & 1,807 \\
\hline
\end{tabular}


Así llegamos a la Tabla 7 y Tabla 8 donde vemos que ninguna variable resulta significativa ya que son mayores de 0,05 , por lo cual no se acepta una posible hipótesis de que el conjunto de variables conformado por: la publicidad de las películas, la crítica, los artistas, las recomendaciones y la cartelera impacten en los gastos mensuales que los espectadores destinan para boletos de entrada al cine, por lo que los espectadores prefieren algunos otros factores, más que los mercadológicos, como bien pudieran ser los factores narrativos: personajes principales, antagonistas y la tensión 0 carga emocional que una película genera en el espectador.

Tabla 7. Coeficientes no estandarizados y estandarizados. Corrido en SPSS

\begin{tabular}{cccccccc}
\hline Modelo & $\begin{array}{c}\text { Beta no } \\
\text { estand. }\end{array}$ & $\begin{array}{c}\text { Error } \\
\text { tip. }\end{array}$ & $\begin{array}{c}\text { Beta } \\
\text { estand. }\end{array}$ & $\mathbf{t}$ & Sig. & $\begin{array}{c}\text { Límite } \\
\text { inferior } \\
95 \%\end{array}$ & $\begin{array}{c}\text { Límite } \\
\text { superior } \\
\mathbf{9 5 \%}\end{array}$ \\
\hline (Constante) & 289.747 & 120.607 & & 2.402 & 0.018 & 50.312 & 529.182 \\
PUBLICIDAD & 17.502 & 12.732 & 0.167 & 1.375 & 0.172 & -7.774 & 42.778 \\
CRÍTICA & 1.315 & 10.407 & 0.015 & 0.126 & 0.900 & -19.346 & 21.975 \\
ARTISTAS & 15.922 & 10.869 & 0.161 & 1.465 & 0.146 & -5.656 & 37.500 \\
RECOMENDACIONES & -20.483 & 14.290 & -0.179 & -1.433 & 0.155 & -48.852 & 7.885 \\
CARTELERA & 5.559 & 11.927 & 0.049 & 0.466 & 0.642 & -18.119 & 29.236 \\
\hline
\end{tabular}

Nota 1: Variable dependiente: GASTO

Tabla 8. Correlaciones y estadísticos de correlación del modelo. Corrido en SPSS

\begin{tabular}{cccccc}
\hline Modelo & $\begin{array}{c}\text { Correlación } \\
\text { Orden cero }\end{array}$ & Parcial & Semiparcial & Tolerancia & FIV \\
\hline (Constante) & & & & & \\
PUBLICIDAD & 0.129 & 0.14 & 0.137 & 0.670 & 1.493 \\
CRÍTICA & 0.070 & 0.013 & 0.013 & 0.713 & 1.402 \\
ARTISTAS & 0.176 & 0.149 & 0.146 & 0.816 & 1.225 \\
RECOMENDACIONES & -0.031 & -0.146 & -0.143 & 0.636 & 1.573 \\
CARTELERA & 0.069 & 0.048 & 0.046 & 0.899 & 1.113 \\
\hline
\end{tabular}

Nota 1: Variable dependiente: GASTO 


\section{Conclusiones}

Es importante que los productores de las OPCM tomen en cuenta las funciones del cine: es un motor cultural para las sociedades y es un motor económico para los países. Estados Unidos se ha caracterizado por ser una nación que utiliza el cine para introducir sus ideas en otros países pero también para generar riquezas por medio de los ingresos en taquillas y de venta de productos adherentes a las películas. Por el contrario, las OPCM no han podido impulsar su producción a cifras que lo ubiquen como uno de los productores más grandes de cine en el mundo. A pesar de que diversas instituciones gubernamentales como CONACULTA, IMCINE, CONARTE, etc. han buscado estimular la producción de películas, las OPCM no han logrado cristalizar estos beneficios en taquilla.

Conocer los diversos factores que conforman el éxito comercial de una película es fundamental para las OPCM, en esta primera parte de la investigación, se analizan si los factores mercadológicos influyen en los espectadores para adquirir un boleto en taquilla y que esto impacte en los ingresos en taquilla de los films. Los factores aquí estudiados son: publicidad, crítica, talento, recomendaciones y disponibilidad en cartelera. Mediante la regresión lineal efectuada se reflejó en la correlación de Pearson, que el gasto no tiene ninguna relación con la publicidad, crítica, talento, recomendación, o disponibilidad en cartelera.

Por medio de la $R$ cuadrado corregida se constató que sólo el 0.01 de la variabilidad en "y" está explicada por este modelo de regresión que mide lo que busca Durbin-Watson (1.807). Por lo tanto, la hipótesis alternativa no se acepta pues ninguna variable independiente resultó significativa para los impactos en los gastos en taquilla (variable dependiente): publicidad (0.172), crítica (0.90), talento (0.146), recomendaciones (0.155) y disponibilidad en cartelera (0.642). Este estudio no pretende persuadir que las producciones nacionales no deban destinar recursos económicos a estas partidas mercadológicas, pues aunque lo anterior refleja que los espectadores toman otros factores para adquirir un boleto en taquilla, como pudieran ser los narrativos: personaje principal, antagonista y tensión (carga emocional) y que dichos elementos será estudiados en la segunda parte de este investigación, los factores mercadológicos informan a los espectadores sobre las opciones que tienen para su consumo de películas generando beneficios a las OPCM. 


\section{Anexo}

Listado de Páginas de internet consultadas

- www.bls.gov

- www.boxofficemojo.com

- www.cinemex.com.mx

- www.cinepolis.com.mx

- www.cinemark.com.mx

- www.conaculta.gob.mx

- www.donniedarkofilm.com

- www.fundeu.es

- www.imcine.gob.mx

- www.inegi.gob.mx

- www.mmcinemas.com.mx

- www.mpaa.org

- www.talentoartistico.com.mx

\section{Referencias}

Brunet, J. (2004). The Social Production of Creative Productos in the Television and Pelicula Industry. Montreal. International Journal of Arts Management, 6(2), $n^{\circ}$ V622.

Cookie, P. (2007). Supporting Contemporary German Films: How Triumphant is the Free Market? Journal of Contemporary European Studies, 15(1), 35-46.

Cusic, D. (2009). Popular Culture and the Economy. Belmont. The Journal of Popular Culture, 42(3), 458-479.

De Vany, A. (2004). Hollywood Economics: How Extreme Uncertainty Shapes the Película Industry, London: Routledge.

Eliashberg, J. et Shugan, S. (1997). Films Critics: Influencers or Predictors? Journal of Marketing, 61(2), 68-78.

Fernández, F. (2008). Manual Básico de Lenguaje y Narrativa Audiovisual. España. Paidos Ibérica.

Gómez, R. (2005). La Industria Cinematográfica Mexicana 1992-2003, Estructura, Desarrollo, Políticas y Tendencias. Colima. Estudio Sobre las Culturas Contemporáneas

González, D. (2008). Hollywood: la genealogía secreta. Monterrey: Universidad Autónoma de Nuevo León.

Haynes, J. (2007). Nollywood in Lagos, Lagos in Nollywood Films. Africa Today, 54(2), 131150. 
Kong, S. (2007). Genre Films, Media Corporations, and the Commercialisation of the Chinese Films Industry: The Case of New Year Comedies. Asian Studies Review, 31(3), 227-242.

Lorenzen, M. (2007). Internationalization vs Globalization of the Films Industry, Industry and Innovation. 14(4), 349 - 357.

Lozano, D., J. Barragán \& S. Guerra. (2009). El Cine: El Negocio de la Cultura. Innovaciones de Negocios. 6(2), 207-224.

Lozano, D., J. Barragán, S. Guerra \& J. Zúñiga. (2010). Estrategias para el Desarrollo Cinematográfico Comercial Mexicano hacia los Estados Unidos. Innovaciones de Negocios, 7(1), 89-104.

Lozano, D., J. Barragán, S. Guerra \& P. Villalpando. (2010). Retos del Cine Mexicano para su Consumo en los Estados Unidos. Innovaciones de Negocios, 7(2), 89-104.

Miguel, J. (2004). Factores de Éxito de las Series y Sagas. Ámbitos, 11/12, 465 - 481.

Morawetz, N. (2007). Finance, Policy and Industrial Dynamics - The Rise of Coproductions in the Film Industry. Industry and Innovation, 14(4), 421-443.

Rosen, S. (2003). China goes Hollywood (review of Ying Zhu, Consumption, Markets, and Culture (2002). Foreign Policy, January-February, (134), 94, 96, 98.

Russell, T. y Lane, R. (2001). Kleppner Publicidad. México: Pearson Educación de México.

Von Dassanowsky, R. (2005). Austrian Cinema: A History. Carolina del Norte. Jefferson, N.C: McFarland \& Co. 